\title{
Angiogenesis inhibitors in gastric and gastroesophageal junction cancer
}

\author{
Giandomenico Roviello ${ }^{1} \cdot$ Roberto Petrioli $^{1} \cdot$ Luigi Marano $^{2} \cdot$ Karol Polom $^{2}$ • \\ Daniele Marrelli ${ }^{3} \cdot$ Armando Perrella $^{1} \cdot$ Franco Roviello $^{2}$
}

Received: 16 April 2015/ Accepted: 15 August 2015/Published online: 2 September 2015

(C) The International Gastric Cancer Association and The Japanese Gastric Cancer Association 2015

\begin{abstract}
Despite significant improvements in systemic chemotherapy during the past two decades, the prognosis of patients with advanced gastric and gastroesophageal junction adenocarcinoma remains poor. Because of molecular heterogeneity, it is essential to classify tumors based on the underlying oncogenic pathways and to develop targeted therapies acting on individual tumors. Unfortunately, although a number of molecular targets have been studied, very few of these agents can be used in a clinical setting. In this review, we summarize the available data on anti-angiogenic agents in advanced/metastatic gastric cancer.
\end{abstract}

Keywords Gastric cancer - VEGF · Bevacizumab · Ramucirumab

\section{Introduction}

Gastric cancer (GC) is a common malignancy and is recognized as the third leading cause of cancer mortality worldwide [1]. Although surgical resection offers the only chance of a long-term cure, the majority of patients with gastric or gastroesophageal junction (GEJ) cancer

Giandomenico Roviello

giandomenicoroviello@hotmail.it

1 Medical Oncology Unit, University of Siena, Viale Bracci 11, 53100 Siena, Italy

2 Unit of General and Minimally Invasive Surgery, Department of Medical, Surgical and Neuroscience, University of Siena, Viale Bracci 11, Siena 53100, Italy

3 Section of Advanced Surgical Oncology, Department of Medical, Surgical and Neurosciences, University of Siena, Viale Bracci 11, Siena 53100, Italy present with locally advanced or metastatic disease at diagnosis. Palliative chemotherapy is the mainstay of treatment for this stage [2,3]. Among chemotherapy agents, cisplatin and oxaliplatin, docetaxel, the fluoropyrimidines 5-fluorouracil and capecitabine, and the anthracyclines epirubicin and doxorubicin are the most active drugs that are conventionally administered in advanced GC or GEJ cancer $[4,5]$. Unfortunately, the outcome of these patients remains poor, with the 5-year survival for advanced or metastatic disease remaining less than $10 \%$ and the median overall survival (OS) limited to 1 year [6]. Therefore, the development of innovative and effective therapies is needed. In response to this need, the recent randomized TOGA trial demonstrated that patients with positive human epidermal receptor 2 (HER-2) tumors may benefit from chemotherapy combined with the monoclonal antibody trastuzumab in terms of progression-free survival (PFS) and OS [7]. However, HER2-overexpressing tumors represent approximately $20 \%$ of the total number of GC [7-9]. Unfortunately, with the exception of trastuzumab in HER2-positive GC, recent studies of other agents targeting oncogenic mediators, such as epidermal growth factor receptor (EGFR), mTOR, and c-Met, have not been shown to improve survival [10-16].

Angiogenesis is one of the main mechanisms for tumor growth and for metastatic dissemination. More recently, the inhibition of angiogenesis has become an area of GC research that has received considerable attention. The aim of this mini-review is to summarize the available data on anti-angiogenic agents in advanced or metastatic GC. 


\section{Angiogenesis}

Vascular endothelial growth factor (VEGF), also known as VEGF-A, is a key regulator of angiogenesis [17]. In patients with GC, circulating VEGF levels are associated with increased tumor aggressiveness and reduced survival [18, 19]. However, the VEGF family consists of other molecules, such as VEGF-B, VEGF-C, VEGF-D, VEGF$\mathrm{E}$, and placental growth factor (PGF). The binding of VEGF to VEGF receptors (VEGFR) regulates the angiogenesis signaling. Each type of VEGF ligand links several VEGFRs, such as VEGFR1 (fms-like tyrosine kinase 1/Flt-1), VEGFR2 (Flk-1/KDR), and VEGFR3 (Flt-4), and two co-receptors, neuropilin 1 and 2 (NRP1/2); in particular, VEGF-A binds to VEGFR-1 and VEGFR-2, VEGF-B and PGF to VEGFR-1, and VEGF-C and D to VEGFR-2 and -3 [20]. Among all receptors, VEGFR2 has a key function in angiogenesis because it is widely considered to be the primary receptor mediating angiogenesis, whereas VEGFR1 and VEGFR3 are classically involved in other pathways, such as monocyte chemotaxis, hematopoietic stem cell survival, and lymphangiogenesis [21]. In animal models of gastric adenocarcinoma, VEGFR-2 inhibition reduced tumor growth and vascularity [22], and the expression of VEGFR2 in intestinaltype GC seems to be a prognostic factor because it is correlated with the vessel count and the stage of disease [23]. In addition, in patients with GC, several studies have investigated the clinical and prognostic relevance of circulating VEGF levels [17, 23-27]. In fact, VEGF levels are associated with increased tumor aggressiveness and reduced survival [17-19]. Interestingly, the role of VEGF appeared to be more relevant in tumors with an intestinaltype rather than a diffuse-type histological architecture [23].

During the past few years, a number of additional complementary angiogenic pathways have been described. These pathways are based on key proteins, such as hypoxia-inducible factor (HIF), platelet-derived growth factor (PDGF), fibroblast growth factor (FGF), angiopoietin (Ang), and Notch, as well as various inflammatory mediators of angiogenesis [28, 29]. Particularly, under hypoxic conditions, HIF- $1 \alpha$ serves as a transcription factor that targets several genes, including VEGF, and promotes an adaptive angiogenic response to hypoxia [30]. In clinical settings, it has been shown that HIF-1 $\alpha$ is associated with tumor prognosis [31-35].

Currently, the most common approaches to inhibiting the VEGF axis include binding of VEGF ligands, small molecular inhibition of receptor tyrosine kinase (RTK) and downstream targets, and steric blockade of VEGFRs (Fig. 1).

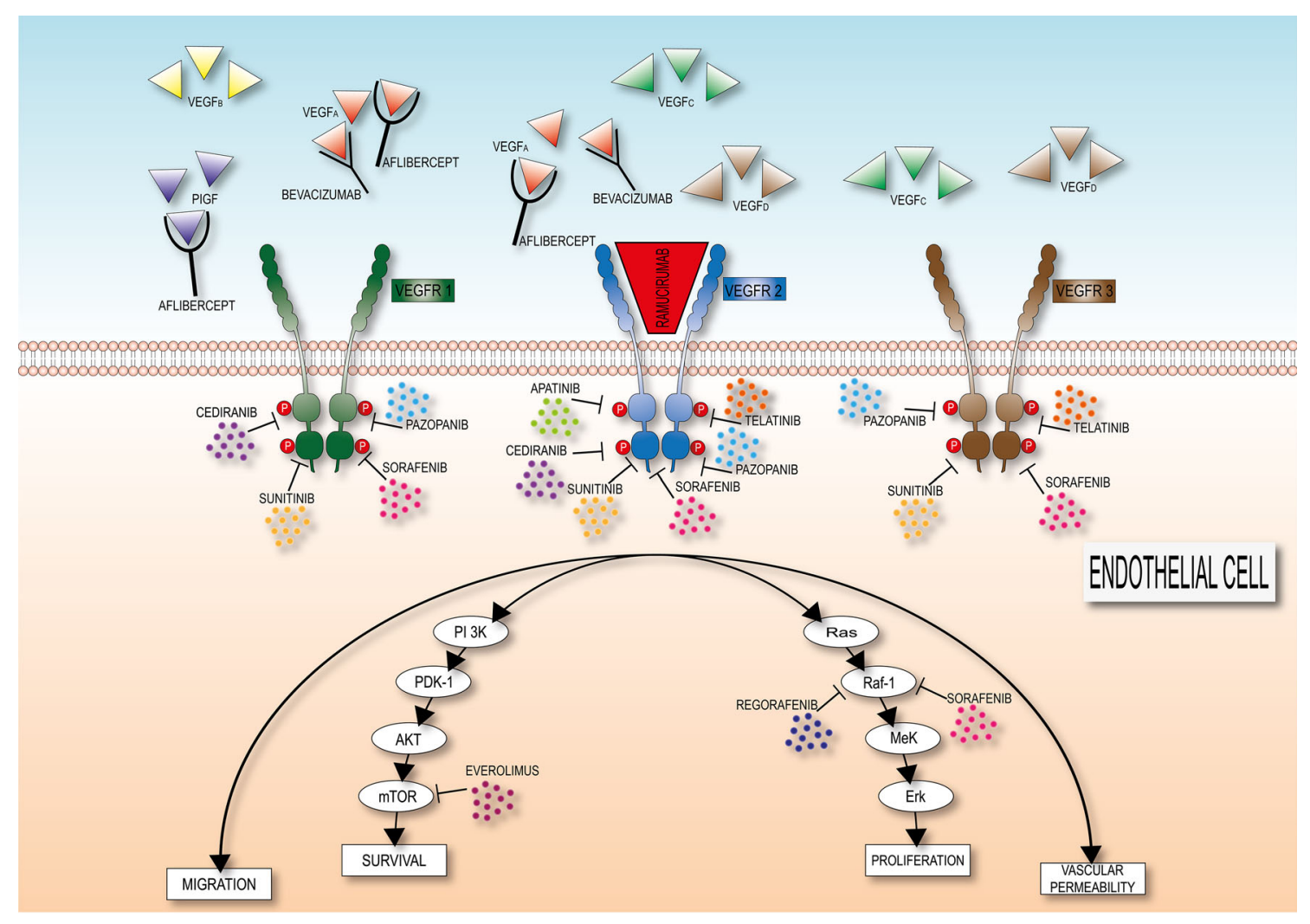

Fig. 1 Mechanisms of action and targets of anti-angiogenic agents that are studied in gastric and gastroesophageal junction cancer 


\section{Bevacizumab}

Bev is a recombinant, humanized, monoclonal antibody against the VEGF. Bevacizumab inhibits angiogenesis by binding to VEGF, blocking its interaction with VEGFR-1 and VEGFR-2. Bevacizumab is currently approved for several cancers, such as colon, lung, breast, ovarian, endometrial, and clear cell renal carcinoma, in a metastatic setting. Four completed phase II and two completed phase III clinical trials have investigated the efficacy of first-line bevacizumab combined with chemotherapy in patients with advanced GC and GEJ tumors.

In 2006, a multicenter phase II study conducted by Shah et al. [36] evaluated the efficacy and safety of the addition of bevacizumab to cisplatin-irinotecan in the first-line setting in 47 patients with metastatic GC and GEJ tumors. Bevacizumab resulted in a response rate (RR) in $65 \%$ of patients, with a median time to progression of 8.3 months and a median OS of 12.3 months. Bevacizumab-related toxicity included a $28 \%$ incidence of grade 3 hypertension, a $25 \%$ incidence of grade 3-4 thromboembolic events, a $4.2 \%$ incidence of gastric perforation, and a $2.1 \%$ incidence of cardiovascular events. Finally, although the primary tumor was unresected in 40 patients, only 1 patient had a significant upper gastrointestinal bleed.

In 2011, another small phase II trial was conducted in metastatic previously untreated patients who had GC and GEJ adenocarcinoma. Bevacizumab, with a modified schedule of docetaxel, cisplatin, and fluorouracil, was investigated in 44 patients [37]. The RR of this combination was $67 \%$; after a 26-month follow-up, the median PFS and OS were 12 months and 16.8 months, respectively. Notably, in subset analysis, diffuse GC had significantly worse PFS and OS. However, no evidence of increased chemotherapy-related toxicities with the addition of bevacizumab was shown. In this trial, $39 \%$ of patients experienced venous thromboembolism.

In contrast to these results, El-Rayes et al. reported less efficacy of the addition of bevacizumab to the chemotherapy regimen with docetaxel and oxaliplatin in 38 previously untreated patients with locally advanced or metastatic disease [38]. The disease control rate (DCR) was $79 \%$ with 6.6 months of PFS and 11.1 months of OS. Finally, Uronis et al. [39] investigated the combination of bevacizumab with capecitabine and oxaliplatin in primary metastatic esophagogastric adenocarcinomas (RR $51 \%$, PFS 7.2 months, OS 10.8 months).

To settle the issue of these discordant results, an international, randomized, double-blind, placebo-controlled phase III trial was conducted: the Avastin for Advanced Gastric Cancer trial (AVAGAST). The trial investigated the combination of bevacizumab with cisplatin and capecitabine in previously untreated unresectable locally advanced or metastatic GC or EGJ adenocarcinomas [40]. Overall survival was the primary endpoint of this study. A total of 774 patients were enrolled; 387 were assigned to each treatment group. The median OS was 12.1 months with bevacizumab plus fluoropyrimidine-cisplatin versus 10.1 months in placebo plus fluoropyrimidine-cisplatin. However, although the bevacizumab-based regimen achieved a median of more than 2 months of OS, this result did not meet the specified criteria for statistical significance (HR $=0.87 ; 95 \%$ CI， 0.73-1.03; $P=0.1002)$. Conversely, both the median PFS (6.7 vs. 5.3 months: $\mathrm{HR}=0.80 ; 95 \% \mathrm{CI}, 0.68-0.93 ; P=0.0037)$ and overall RR (46.0\% vs. $37.4 \%$ : HR $=8.61 ; 95 \%$ CI, 0.6-16.6; $P=0.0315)$ were significantly improved with bevacizumab versus placebo. Among the bevacizumab-related adverse events, with the exception of the incidence of grade $\geq 3$ hypertension ( $6 \%$ vs. $<1 \%$ ), no increased incidence of other grade $\geq 3$ adverse events of special interest for bevacizumab were observed in the experimental arm compared to the placebo arm. Interestingly, the preplanned subgroup analyses revealed regional differences in efficacy outcomes. Indeed, the survival benefit of bevacizumab was substantially higher in patients enrolled in North America and Latin America, whereas patients enrolled in Asia appeared to have no benefit, and European patients had intermediate results. The authors try to explain this fact by citing the diversity of patient selection, clinical practice, population genetics, and second-line chemotherapy among all patients.

Subsequently, a smaller phase III study, which mimicked the design of the AVAGAST trial, was conducted in 202 Chinese patients. Therefore, in the AVATAR trial [41], the baseline patient clinical characteristics and the second line of treatment were more similar to the European-American subgroup than the Asian Pacific subgroup of the AVAGAST trial. However, this study showed no significant differences between chemotherapy plus bevacizumab versus chemotherapy (OS: 10.5 months vs. 11.4 months; HR $=1.11 ; 95 \%$ CI, 0.79-1.56; $P=0.56$; PFS: 6.3 months vs. 6.0 months; $\mathrm{HR}=0.89 ; 95 \% \mathrm{CI}$, $0.66-1.21 ; P=0.47)$. In this trial, both the incidence of grade $\geq 3$ adverse events and the incidence of grade $\geq 3$ adverse events of special interest for bevacizumab were similar between the two treatment arms.

Finally, ST03 was a multicenter, randomized, phase II/ III study comparing perioperative epirubicin, cisplatin, and capecitabine (ECX) with or without bevacizumab and was designed with the first 200 patients contributing to a phase II assessment of safety to exclude unacceptable rates of gastrointestinal perforation and cardiotoxicity. In this interim analysis, the incidence of cardiac complications 
was similar in both arms, except for arterial thromboembolic events and more asymptomatic left ventricular ejection fraction falls, which were more frequent with ECX plus bevacizumab [42]. However, the esophageal stratum was closed prematurely because of safety concerns. Some hospitals that are taking part in the ST03 trial are also participating in the ST03 lapatinib feasibility study. The patients with human epidermal growth factor receptor-2 (HER-2) positivity were randomized to receive ECX chemotherapy alone against modified ECX with lapatinib. To date, the study is currently recruiting participants (NCT00450203).

Based on the reported results, bevacizumab cannot currently be considered an option for GC patients with unresectable or metastatic tumors. The results of published phase III trials with bevacizumab in GC are summarized in Table 1.

\section{Ramucirumab}

Ramucirumab, a human IgG1 monoclonal antibody against VEGFR-2, prevents ligand binding and receptor-mediated pathway activation in endothelial cells [43]. Preclinical study results highlighted that blocking this VEGF family receptor was associated with the inhibition of VEGF-mediated signaling, proliferation and migration of human endothelial cells, and antitumor activity in animal models [43-47]. More recently, two phase III clinical trials have shown that ramucirumab is a valuable therapeutic option in GC (Table 2).

The first trial was an international, randomized, doubleblind, placebo-controlled, phase III trial involving patients with advanced GC or GEJ adenocarcinoma and disease progression after first-line platinum-containing or fluoropyrimidine-containing chemotherapy (REGARD) [48].

Table 1 Phase III clinical trails of bevacizumab in gastric and gastroesophageal junction cancer

\begin{tabular}{|c|c|c|c|c|c|c|c|}
\hline Authors & Setting & $\begin{array}{l}\text { Patients } \\
(n)\end{array}$ & Regimen & End points & ORR & TTP/PFS (months) & OS (months) \\
\hline \multirow[t]{2}{*}{$\begin{array}{l}\text { Ohtsu et al. } \\
\text { (AVAGAST) }\end{array}$} & \multirow[t]{2}{*}{$\begin{array}{l}\text { 1st } \\
\text { line }\end{array}$} & 387 & $\mathrm{XC}+$ bev & $\begin{array}{l}\text { Primary: } \\
\text { OS }\end{array}$ & $46 \%$ (bev) & (6.7) bev & (12.1) bev \\
\hline & & 387 & $\mathrm{XC}+$ placebo & $\begin{array}{l}\text { Secondary: } \\
\text { PFS, } \\
\text { ORR, } \\
\text { safety }\end{array}$ & $\begin{array}{l}37.4 \% \text { (placebo) (HR } \\
8.61 ; 95 \% \text { CI } \\
0.6-16.6 \\
P=0.0315)\end{array}$ & $\begin{array}{l}\text { (5.3) placebo (HR } \\
0.80 ; 95 \% \text { CI } \\
0.68-0.93 ; \\
P=0.0037)\end{array}$ & $\begin{array}{l}\text { (10.1) placebo (HR } \\
0.87 ; 95 \% \text { CI } \\
0.73-1.03 ; \\
P=0.1002)\end{array}$ \\
\hline \multirow[t]{2}{*}{$\begin{array}{l}\text { Shen et al. } \\
\text { (AVATAR) }\end{array}$} & \multirow[t]{2}{*}{$\begin{array}{l}\text { 1st } \\
\text { line }\end{array}$} & 100 & $\mathrm{XC}+\mathrm{bev}$ & $\begin{array}{l}\text { Primary: } \\
\text { OS }\end{array}$ & $41 \%$ (bev) & (6.3) bev & (10.5) bev \\
\hline & & 102 & $\mathrm{XC}+$ placebo & $\begin{array}{l}\text { Secondary: } \\
\text { PFS, } \\
\text { ORR }\end{array}$ & $\begin{array}{c}34 \% \text { (placebo) }(\mathrm{HR} \\
7.02 ; 95 \% \mathrm{CI}-8.3 \\
\text { to } 22.4 ; P=0.34)\end{array}$ & $\begin{array}{l}\text { (6.0) placebo }(\mathrm{HR} \\
0.89 ; 95 \% \text { CI } \\
0.66-1.21 \\
P=0.47)\end{array}$ & $\begin{array}{l}\text { (11.4) placebo }(\mathrm{HR} \\
1.11 ; 95 \% \mathrm{CI} \\
0.79-1.56 \\
P=0.56)\end{array}$ \\
\hline
\end{tabular}

ORR median overall response rate, TTP median time to progression, $P F S$ median progression-free survival, $O S$ median overall survival, $X$ capecitabine, $C$ cisplatin, $B e v$ bevacizumab, $H R$ hazard ratio, $C I$ confidence interval

Table 2 Phase III clinical trails of ramucirumab in gastric and gastroesophageal junction cancer

\begin{tabular}{|c|c|c|c|c|c|c|c|}
\hline Authors & Setting & $\begin{array}{l}\text { Patients } \\
(n)\end{array}$ & Regimen & End points & ORR & PFS (months) & OS (months) \\
\hline \multirow{2}{*}{$\begin{array}{l}\text { Fuchs et al. } \\
\text { (REGARD) }\end{array}$} & \multirow{2}{*}{$\begin{array}{l}\text { 2nd } \\
\text { line }\end{array}$} & 238 & $\mathrm{Ram}+\mathrm{BSC}$ & Primary: OS & $3 \%$ (ram) & (2.1) ram & (5.2) ram \\
\hline & & 117 & $\mathrm{BSC}$ & $\begin{array}{l}\text { Secondary: PFS, rate of } \\
12 \text { weeks; PFS, } \\
\text { duration of disease } \\
\text { control }\end{array}$ & $\begin{array}{l}3 \% \\
\text { (placebo) }\end{array}$ & $\begin{array}{l}\text { (1.3) placebo (HR } \\
0.483 ; 95 \% \text { CI } \\
0.376-0.620 \\
P=0.0001)\end{array}$ & $\begin{array}{l}\text { (3.8) placebo (HR } \\
0.776 ; 95 \% \mathrm{CI} \\
0.603-0.998 \\
P=0.047)\end{array}$ \\
\hline \multirow[t]{2}{*}{$\begin{array}{l}\text { Wilke et al. } \\
\text { (RAINBOW) }\end{array}$} & \multirow[t]{2}{*}{$\begin{array}{l}\text { 2nd } \\
\text { line }\end{array}$} & 330 & PTX + Ram & Primary: OS & $\begin{array}{l}28 \% \\
(\mathrm{ram})\end{array}$ & (4.40) ram & (9.63) ram \\
\hline & & 335 & PTX + placebo & Secondary: PFS, ORR & $\begin{array}{l}16 \% \\
\text { (placebo) }\end{array}$ & $\begin{array}{l}\text { (2.86) Placebo (HR } \\
0.635 ; 95 \% \text { CI } \\
0.536-0.752 ; \\
P=0.0001)\end{array}$ & $\begin{array}{l}\text { (7.26) placebo (HR } \\
0.807 ; 95 \% \text { CI } \\
0.678-0.962 ; \\
P=0.0169)\end{array}$ \\
\hline
\end{tabular}

ORR median overall response rate, $P F S$ median progression-free survival, $O S$ median overall survival, Ram ramucirumab, $B S C$ best supportive care, $H R$ hazard ratio, $C I$ confidence interval, PTX paclitaxel 
The patients of this study were randomly assigned (2:1) to receive the best supportive care plus either ramucirumab or placebo. The primary endpoint was OS. A total of 355 patients were assigned to receive ramucirumab $(n=238)$ or placebo $(n=117)$. The median OS was 5.2 months in patients in the ramucirumab group and 3.8 months in those in the placebo group ( $\mathrm{HR}=0.776$; $95 \% \mathrm{CI}, 0.603-0.998$; $P=0.047)$. For PFS (2.1 months vs. 1.3 months with placebo: HR 0.483; $95 \%$ CI, 0.376-0.620; $P<0.0001$ ), the rate of disease control was significantly higher in patients given ramucirumab than in those given placebo (49\% vs. $23 \% ; P<0.0001$ ). The rates of serious adverse events were similar between the two arms. However, the rates of hypertension were higher in the ramucirumab group than in the placebo group [38 (16\%) vs. $9(8 \%)$ ], whereas the rates of other adverse events were mostly similar between groups [223 (94\%) vs. $101(88 \%)]$. The effect of ramucirumab on quality of life was observed in patients who received at least four cycles of therapy with ramucirumab, and the performance status was also maintained for a significantly longer time with ramucirumab. Although no regional differences in the effects of ramucirumab were reported, in this study, the number of Asian patients $(16 \%)$ or patients recruited in Asian centers $(7 \%)$ is too small to make definitive conclusions.

In 2014, the results of the RAINBOW trial were reported. The RAINBOW trial is a randomized, placebocontrolled, double-blind, phase III trial that investigated ramucirumab or placebo plus paclitaxel in patients with advanced GC or GEJ adenocarcinoma and disease progression on or within 4 months after first-line chemotherapy (platinum plus fluoropyrimidine with or without an anthracycline) [49]. The randomization was 1:1. The primary endpoint was OS. Six hundred sixty-five patients were randomly assigned to treatment, 330 to ramucirumab plus paclitaxel and 335 to placebo plus paclitaxel. Overall survival was significantly longer in the ramucirumab plus paclitaxel group than in the placebo plus paclitaxel group (median, 9.6 vs. 7.4 months: $\mathrm{HR}=0.807 ; 95 \% \mathrm{CI}$, $0.678-0.962 ; P=0.0169)$. The study also met its secondary endpoints of PFS (2.86 vs. 4.40 months: $\mathrm{HR}=0.635 ; 95 \% \mathrm{CI}, 0.536-0.752 ; P=0.0001)$ and RR (16\% vs. $28 \% ; P=0.0001)$. At 6 months, the PFS rate was $36 \%$ versus $17 \%$ and at 9 months was $22 \%$ versus $10 \%$. When survival outcomes are analyzed by geographic region, ramucirumab seems to have similar activity in both Asian patients (33.5\% of the study population) and Western patients $(66.5 \%$ of the study population) [49]. Ramucirumab was relatively well tolerated, although adverse events of grade $\geq 3$ were somewhat greater with combination treatment and included neutropenia (40.7\% vs. $18.8 \%$ ); however, the incidence of febrile neutropenia was comparable (3.1\% vs. $2.4 \%)$, as were hypertension (14.1\% vs. $2.4 \%)$ and fatigue $(7.0 \%$ vs. $4 . \%)$.

Based on the results of REGARD, in April 2014, ramucirumab was granted FDA approval as a second-line treatment in patients with advanced or metastatic GC or GEJ cancers who progressed on fluoropyrimidine-or platinum-containing first-line chemotherapy. In November 2014, the FDA approved the combination of ramucirumab and paclitaxel as a treatment for patients with previously treated advanced gastric or gastroesophageal junction (GEJ) adenocarcinoma based on data from the phase III RAINBOW trial. In Europe, since December 2014, the EMA has approved ramucirumab in combination with paclitaxel for the treatment of patients with advanced GC or EGJ adenocarcinoma with disease progression after prior platinum or fluoropyrimidine chemotherapy. In Asian countries, ramucirumab is undertaken as a second line of treatment.

Unfortunately, the preliminary results have shown that ramucirumab failed to achieve superiority over chemotherapy alone in a first-line setting. In a doubleblind, phase II trial, 168 patients with previously untreated unresectable locally advanced or metastatic esophageal, $\mathrm{GC}$, or EGJ adenocarcinoma were randomized to receive mFOLFOX6 plus ramucirumab or placebo [50]. One hundred sixty-eight patients were enrolled ( 84 in the experimental arm vs. 84 in the chemotherapy + placebo arm). PFS (primary endpoint) and OS were similar (6.4 vs. 6.7 months: HR $0.98 ; 95 \% \mathrm{CI}, 0.69-1.37 ; P=0.89)$ and OS (11.7 vs. 11.5 months: HR 1.08) between the two arms. Subgroup analyses by primary tumor location showed that for esophageal adenocarcinoma the median PFS was 5.6 versus 6.1 months; for GC or GEJ adenocarcinoma, the PFS was 8.7 versus 7.1 months and OS 14.6 versus 12.5 months. The PFS rate at 3 months was higher in the experimental arm ( $89 \%$ vs. $75 \% ; P=0.020)$, but not at 6,9 , or 12 months. Subgroup analyses suggest that the negative results of this study may be explained by the high rate of esophageal cancers $(>45 \%)$ in the study's population and the higher rate of treatment discontinuation before tumor progression in the experimental arm (27\% vs. $10 \%$ ).

In conclusion, ramucirumab is now recommended in second-line treatment for GC. Particularly, for patients with ECOG 0-1, ramucirumab can be administered in combination with pacliatexel, whereas for patients with ECOG 0-2, ramucirumab can be administered in monotherapy without taxane. Finally, for patients with an ECOG status $\geq 2$, the benefit from second-line treatment with ramucirumab remains unclear [51]. 


\section{TKI inhibitor}

\section{Sorafenib}

Sorafenib is an oral multi-target TKI inhibitor linked to VEGFR-1, VEGFR-2, VEGFR-3, platelet-derived growth factor receptor (PDGFR), B-Raf, Raf-1, and c-Kit. The main antineoplastic activity of sorafenib is obtained by the inhibition of tumor proliferation by blocking the RAF/ MEK/ERK-mediated cell signaling pathway. However, although it is mediated by blocking VEGFR and PDGFR, sorafenib shows anti-angiogenic activity [52]. The ECOG 5203 phase II trial [53] evaluated the efficacy and toxicity of combined sorafenib, docetaxel, and cisplatin in patients with metastatic or advanced GC or GEJ adenocarcinoma. Forty-four chemotherapy-naïve patients were enrolled: they achieved an overall RR of $41 \%$ (primary endpoint), an OS of 13.6 months, and a PFS of 5.8 months. The major toxicity of this regimen was neutropenia, which reached grade 3 to 4 in $64 \%$ of patients. One patient experienced hemorrhage at the tumor site. Another phase II study was performed to assess the combination of oxaliplatin and sorafenib as a second-line therapy for GC after treatment with cisplatin and fluoropyrimidine first-line therapy. Among 40 patients, the DCR was approximately $50 \%$. The median PFS was 3 months, and the median OS was 6.5 months [54]. Grade 3-4 neutropenia (9.8\%), thrombocytopenia $(7.3 \%)$, and neurotoxicity $(4.9 \%)$ were reported. The association of sorafenib and oxliplatin resulted in a good safety profile.

Recently, during ESMO 2014, the results of the STARGATE trial comparing capecitabine plus cisplatin with capecitabine plus cisplatin with sorafenib were reported [55]. The primary endpoint of this study was PFS, and a total of 195 patients were randomized. The overall RR was $54 \%$ in the experimental arm and $52 \%$ in the chemotherapy arm $(P=0.826)$. The median PFS assessed by independent review was 5.6 months in sorafenib \pm chemotherapy and 5.3 months in the chemotherapy-alone arm (HR 0.92; $95 \%$ CI, 0.67-1.27; $P=0.609$ ). There was no difference in OS between the two arms (median 11.7 vs. 10.8 months: HR 0.93; $95 \%$ CI, $0.65-1.31 ; P=0.661)$. In this study, the addition of sorafenib to chemotherapy was safe but not more effective than chemotherapy alone.

\section{Sunitinib}

Sunitinib suppresses PDGFR, Kit, rearranged during transfection (RET), Flt-3, and VEGFR-1, VEGFR-2, and VEGFR-3. A phase II study tested single-agent sunitinib in
78 patients as the second line of therapy. The primary endpoint was the overall RR. Two patients $(2.6 \%)$ had partial responses, whereas 25 patients $(32.1 \%)$ had stable disease. The median PFS was 2.3 months, and the median OS was 6.8 months. Grade 3-4 thrombocytopenia and neutropenia were reported in $34.6 \%$ and $29.4 \%$ of patients, respectively [56]. In another phase II trial, 52 patients with chemo-resistant advanced GC received sunitinib as a single agent, obtaining a median OS of 5.8 months [57]. These results do not support the role of single-agent sunitinib therapy in the second line of treatment for GC, and no further clinical trials in GC were launched.

\section{Apatinib}

Apatinib is a TKI selectively targeting VEGFR-2 [58, 59]. A phase I clinical trial showed that this agent has antitumor activity in Chinese patients with metastatic GC [60]. Subsequently, a phase II, randomized, double-blind, placebo-controlled trial was conducted. This trial investigated the safety and efficacy of apatinib as a treatment option for heavily pretreated patients with metastatic GC. One hundred forty-four patients were enrolled to receive placebo (group A), apatinib $850 \mathrm{mg}$ once daily (group B), or apatinib $425 \mathrm{mg}$ twice daily (group C). In groups $\mathrm{A}, \mathrm{B}$, and C, the median OS was $2.50,4.83$, and 4.27 months, respectively, and the median PFS was 1.40, 3.67, and 3.20 months, respectively. The toxicities were tolerable or could be clinically managed. Therefore, the dosing regimen of $850 \mathrm{mg}$ once daily was recommended for following studies [61]. A third-line setting, randomized, phase III trial is currently comparing apatinib $(850 \mathrm{mg} /$ daily) to placebo. Overall survival (OS) is the primary endpoint, and PFS, DCR, overall RR, quality of life, and safety profile are the secondary endpoints [62]. The preliminary results of this study reported a median OS significantly longer in the apatinib group compared with that in the placebo group (195 days versus 140 days: $\mathrm{HR}=0.71 ; 95 \% \quad \mathrm{CI}$, $0.54-0.94 ; P<0.016)$. The median PFS was also prolonged in the apatinib group compared with the placebo group (78 days versus 53: $\mathrm{HR}=0.44 ; 95 \% \mathrm{CI}, 0.33-0.61$; $P<0.0001)$. The objective RR of the apatinib group and placebo group were $2.84 \%$ and $0.00 \%$, respectively. In regard to adverse events, treatment of the apatinib group was generally well tolerated. Most of the adverse reactions could be managed by dose interruptions or reductions. The grade $3 / 4$ adverse reactions that occurred in more than $2 \%$ of patients were hypertension, hand-and-foot syndrome, proteinuria, fatigue, anorexia, and elevated aminotransferase. 


\section{Cediranib}

Cediranib (AZD2171) is a VEGFR-1 and VEGFR-2, c-Kit, and PDGFR- $\beta$ inhibitor [63]. A phase I trial evaluated the association of cediranib with cisplatin plus S-1 or capecitabine. Fourteen untreated advanced GC patients were enrolled. Although a safety profile has emerged, the response seemed modest (only one CR and three PR). Therefore, results from additional studies are needed [64].

\section{Telatinib}

Telatinib is an oral selective inhibitor of VEGFR (VEGFR2, VEGFR-3), PDGFR, and KIT tyrosine kinases. Telatinib associated with standard chemotherapy was tested in 39 untreated patients with GC in a phase II trial. The primary outcome was PFS, and the secondary outcomes were OS, ORR, safety, and tolerability, pharmacokinetic (PK), and biomarkers. A $92 \%$ DCR and 140-day PFS were detected; the association was well tolerated. The drug has shown a good toxicity profile [65].

\section{Other VEGF inhibitors}

Other VEGF-targeted therapies are currently being studied. Two phase II randomized with placebo trials are ongoing using pazopanib in combination with capecitabine plus oxaliplatin (NCT01130805) or in combination with fluorouracil, leucovorin, and oxaliplatin (NCT01503372) as the first-line setting. Aflibercept (NCT01747551) is under investigation in the same setting of patients in combination with folinic acid-fluorouraciloxaliplatin-based chemotherapy. All these studies are currently recruiting participants. More recently, during ASCO 2015, the results of a phase II randomized trial of regorafenib (an oral multi-kinase inhibitor with anti-angiogenic property) were reported [66]. Patients with GC who progressed after a first and second line of therapy were randomized to receive regorafenib or placebo. The primary endpoint was PFS. A total of 152 patients were enrolled, yielding 147 evaluable for analysis (97 regorafenib and 50 placebo). The median PFS was 11.1 weeks and 3.9 weeks for regorafenib and placebo, respectively $(P<0.0001)$; the median OS was 25 weeks and 19.4 weeks, respectively $(P=0.11)$. Prespecified analyses found that regorafenib had a greater effect in Korea than in Australia and New Zealand (HR 0.12 vs. 0.61; $P=0.0009$ ). Regorafenib was well tolerated, with the expected spectrum of toxicities.

\section{PI3K-AKT-MTOR inhibitor}

The PI3K/AKT pathway is an intracellular signaling pathway that is important in angiogenesis [67]. Everolimus (RAD001) is an oral inhibitor of the mammalian target of rapamycin serine-threonine kinase (mTOR), inhibiting the PI3K/Akt/mTOR pathway. In 2013, an international, double-blind, phase III study compared the efficacy and safety of everolimus with the best supportive care in previously treated advanced GC (GRANITE-1) [68]. Patients with advanced GC that progressed after one or two lines of systemic chemotherapy were randomly assigned to everolimus $10 \mathrm{mg} /$ day or placebo, both of which groups were given the best supportive care. The primary endpoint was OS. Six hundred fifty-six patients were enrolled. The median OS was 5.4 months with everolimus and 4.3 months with placebo (HR 0.90; $95 \%$ CI, 0.75 to 1.08 ; $P=0.124)$. The median PFS was 1.7 and 1.4 months in the everolimus and placebo arms, respectively (HR 0.66; $95 \%$ CI, 0.56 to $0.78 ; P<0.001)$. However, formal statistical significance could not be declared per the hierarchical testing strategy. The safety profile observed for everolimus was consistent with that observed for everolimus in other cancers. Compared with best supportive care, everolimus did not significantly improve OS for advanced GC that progressed after one or two lines of previous systemic chemotherapy.

\section{Biomarkers}

To date, no predictive biomarkers are available to predict the benefit of anti-angiogenic agents in the treatment of solid tumors. Therefore, it is difficult to identify patients who are more sensitive to inhibitors of angiogenesis. In $\mathrm{GC}$, data on the role of potential biomarkers are available only for bevacizumab and are derived from the preplanned correlative analyses of the AVAGAST trial [69]. In this study, blood and tumor tissue samples of patients treated with bevacizumab or placebo in combination with chemotherapy were collected at baseline. Prespecified biomarkers included plasma VEGF-A and protein expression of neuropilin-1, VEGFR-1, and VEGFR-2. Correlations between biomarkers and clinical outcomes were assessed using a Cox proportional hazards model. Baseline plasma VEGF-A levels and tumor neuropilin-1 expression were identified as potential predictors of bevacizumab efficacy. Patients with high baseline plasma VEGF-A levels showed a trend toward improved OS (HR 0.72; $95 \%$ CI, 0.57-0.93) versus patients with low VEGF-A levels (HR 1.01; $95 \% \mathrm{CI}, 0.77-1.31$; interaction $P=0.07$ ). Patients with low baseline expression of neuropilin-1 also 
showed a trend toward improved OS (HR 0.75; $95 \%$ CI, 0.59-0.97) versus patients with high neuropilin-1 expression (HR 1.07; $95 \%$ CI, 0.81-1.40; interaction $P=0.06$ ). For both biomarkers, subgroup analyses demonstrated significance only in patients from non-Asian regions. Finally, an exploratory analysis of a phase II trial of bevacizumab in combination with capecitabine and oxaliplatin showed that tumor expression of NRP-1 and NRP-2 was assessed and correlated with outcome [39]. However, the small sample size and the absence of a control group do not allow for any conclusions to be drawn regarding the predictive effect of these biomarkers. Therefore, to date, no biomarkers of response are available in a clinical setting.

\section{Discussion}

Angiogenesis has a major function in tumor development and progression. In this setting, clinical data suggest that targeting angiogenesis by inhibiting angiogenic signaling pathways is an important therapeutic activity for many solid tumors. However, evidence of antitumor activity with anti-angiogenic therapies leading to improved OS or PFS in patients with metastatic GC is still limited [70, 71]. Several factors, including heterogeneity of patient populations, ethnic differences, drug mechanisms of action, combination with chemotherapy, and study design, may explain the variable results observed with these agents in the clinical setting. Therefore, because the expression of different and potential targets depends on the tumor site, histology, and ethnic differences, it seems to be important to design clinical trials that are stratified according to these factors [72]. Another key reason for unobserved heterogeneity in response to treatments might be related to insufficient attention to the underlying molecular mechanisms driving differences in cancer aggressiveness and treatment outcomes [73]. Additionally, the lack of predictive biomarkers does not allow for an appropriate selection of patients. Finally, the small effect of anti-angiogenic agents on the OS of GC or GEJ patients underscores how the results of phase II trials are limited when we translated them into phase III trials in a clinical setting. For the majority of molecules, promising phase II trials failed during subsequent phase III trials. Therefore, more caution is needed before starting a phase III trial from the data of a small phase II trial.

In our review, we summarize the available data as well as the current status of ongoing randomized studies on the anti-angiogenic agents in advanced/metastatic GC. To date, the only anti-antiangiogenic agent approved for GC or EGJ patients is ramucirumab. This drug is approved for previously treated patients with advanced or metastatic GC or GEJ adenocarcinoma initially as monotherapy and subsequently as combination therapy with paclitaxel. Subgroup analyses on differential treatment effects between ethnic subgroups have also generated confusion [51]. For example, in the RAINBOW trial [49], the results of a preplanned subgroup analysis showed an advantage (albeit not statistically significant) in the median OS for ramucirumab plus paclitaxel between Asian and non-Asian patients. However, the cohort of Asian patients (especially Japanese patients) more commonly received second and further lines of therapy $[40,51]$. Approximately $70 \%$ of Asian patients and almost $35 \%$ of European and American patients received a post-discontinuation treatment in the RAINBOW trial. Therefore, this fact may explain the differences in OS among Asian and non-Asian regions.

Another important issue pertains to the chemotherapy combination with antiangiogenic treatment. To date, the combination of ramucirumab and paclitaxel is the only combination that has reported positive results in OS. In this setting, a phase II study (NCT02317991) is currently recruiting patients to show whether nab-paclitaxel (an less toxic albumin-based formulation of paclitaxel approved for treatment of various cancers) and ramucirumab are effective when used in combination for treating patients with metastatic gastroesophageal cancer in the second line of treatment. Conversely, combination therapies between platinum-based chemotherapy and anti-angiogenic agents in first-line treatment of GC have failed to show significant survival benefit $[40,41,51]$. Additionally, the first report on ramucirumab in combination with a platinum compound, such as oxaliplatin, did not meet its primary endpoint [50]. Therefore, a randomized, double-blind, placebo-controlled phase III study of cisplatin plus a fluoropyrimidine with or without ramucirumab as first-line therapy in patients with metastatic GC or GEJ adenocarcinoma (RAINFALL, NCT02314117) is currently recruiting participants, and the final results of this trial are awaited to make definitive conclusions about platinumbased chemotherapy and antiangiogenic agents in first-line treatment of GC.

Finally, although the continuation of an anti-angiogenic agent beyond progression could be particularly intriguing, no data are available about the use of ramucirumab in this particular condition for GC patients.

Nonetheless, ramucirumab and apatinib have generated positive results at this time. Recent acquisitions suggest that angiogenesis inhibition is the correct path. In fact, the investigators from the Cancer Genome Atlas Research Network have provided a comprehensive molecular characterization of GC [74]. The scientists propose a molecular classification dividing GC into four subtypes: tumors positive for Epstein-Barr virus; microsatellite unstable tumors; genomically stable tumors; and tumors with chromosomal instability. Notably, in two of these subtypes, 
chromosomally unstable tumors and genomically stable tumors were associated with recurrent amplification of the VEGF-A gene and elevated expression of the angiogenesisrelated pathway [70, 71]. Although these preclinical data are not yet translated into clinical routine practice, it is indisputable that these data provide support for further trials on anti-angiogenic therapy.

In conclusion, targeting the angiogenic pathway has significant clinical potential in GC, even if mounting preclinical evidence indicates that the development of antiVEGF pathway inhibitors may require a deep knowledge on how and to what extent tumors are VEGF addicted through ligand-dependent and ligand-independent mechanisms. The strategy of combining treatments targeting several molecules, or drugs blocking downstream signaling transducers, might be used to address the common problem of crosstalk between signaling pathways and thus the development of resistance. This finding points to the urgent need of high-quality well-designed clinical trials with extensive translational research.

Acknowledgments We thank Giuseppe Guida for the help given in the creation and development of Fig. 1.

\section{Compliance with ethical standards}

Conflict of interest The authors declare that they have no conflict of interest.

\section{References}

1. Torre LA, Bray F, Siegel RL, Ferlay J, Lortet-Tieulent J, Jemal A. Global cancer statistics, 2012. CA Cancer J Clin. 2015;65(2):87-108.

2. Buzzoni R, Bajetta E, Di Bartolomeo M, Miceli R, Beretta E, Ferrario E, et al. Pathological features as predictors of recurrence after radical resection of gastric cancer. $\mathrm{Br} \mathrm{J}$ Surg. 2006;93:205-9.

3. Field K, Michael M, Leong T. Locally advanced and metastatic gastric cancer: current management and new treatment developments. Drugs. 2008;68:299-317.

4. Wagner AD, Unverzagt S, Grothe W, Kleber G, Grothey A, Haerting J, et al. Chemotherapy for advanced gastric cancer. Cochrane Database Syst Rev. 2010;3:CD004064.

5. Petrioli R, Francini E, Roviello F, Marrelli D, Fiaschi AI, Laera L, Rossi G, Bianco V, Brozzetti S, Roviello G. Sequential treatment with epirubicin, oxaliplatin and 5FU (EOF) followed by docetaxel, oxaliplatin and $5 \mathrm{FU}$ (DOF) in patients with advanced gastric or gastroesophageal cancer: a single-institution experience. Cancer Chemother Pharmacol. 2015;75(5):941-7.

6. Lordick F, Allum W, Carneiro F, Mitry E, Tabernero J, Tan P, Van Cutsem E, van de Velde C, Cervantes A. Unmet needs and challenges in gastric cancer: the way forward. Cancer Treat Rev. 2014;40(6):692-700.

7. Bang YJ, Van Cutsem E, Feyereislova A, Chung HC, Shen L, Sawaki A, et al. Trastuzumab in combination with chemotherapy versus chemotherapy alone for treatment of HER2-positive advanced gastric or gastro-oesophageal junction cancer (ToGA): a phase 3, open-label, randomised controlled trial. Lancet. 2010;376(9742):687-97.

8. de Mello RA, Marques AM, Araújo A. HER2 therapies and gastric cancer: a step forward. World J Gastroenterol. 2013;19:6165-9. doi:10.3748/wjg.v19.i37.6165.

9. Janjigian YY, Werner D, Pauligk C, et al. Prognosis of metastatic gastric and gastroesophageal junction cancer by HER2 status: a European and USA International collaborative analysis. Ann Oncol. 2012;23:2656-62.

10. Deng N, Goh LK, Wang H, et al. A comprehensive survey of genomic alterations in gastric cancer reveals systematic patterns of molecular exclusivity and co-occurrence among distinct therapeutic targets. Gut. 2012;61:673-84. doi:10.1136/gutjnl-2011301839.

11. Okines AF, Ashley SE, Cunningham D, et al. Epirubicin, oxaliplatin, and capecitabine with or without panitumumab for advanced esophagogastric cancer: dose-finding study for the prospective multicenter, randomized, phase II/III REAL-3 trial. J Clin Oncol. 2010;28:3945-50.

12. Rao S, Starling N, Cunningham D, et al. Phase I study of epirubicin, cisplatin and capecitabine plus matuzumab in previously untreated patients with advanced oesophagogastric cancer. Br J Cancer. 2008;99:868-74.

13. Rojo F, Tabernero J, Albanell J, et al. Pharmacodynamic studies of gefitinib in tumor biopsy specimens from patients with advanced gastric carcinoma. J Clin Oncol. 2006;24:4309-16.

14. Dragovich T, McCoy S, Fenoglio-Preiser CM, et al. Phase II trial of erlotinib in gastroesophageal junction and gastric adenocarcinomas: SWOG0127. J Clin Oncol. 2006;24:4922-7.

15. Doi T, Muro K, Boku N, et al. Multicenter phase II study of everolimus in patients with previously treated metastatic gastric cancer. J Clin Oncol. 2010;28:1904-10.

16. Marano L, Chiari R, Fabozzi A, De Vita F, Boccardi V, Roviello G, Petrioli R, Marrelli D, Roviello F, Patriti A. c-Met targeting in advanced gastric cancer: an open challenge. Cancer Lett. 2015;365(1):30-6.

17. Maeda K, Chung YS, Ogawa Y, Takatsuka S, Kang SM, Ogawa M, Sawada T, Sowa M. Prognostic value of vascular endothelial growth factor expression in gastric carcinoma. Cancer (Phila). 1996;77:858-63.

18. Jüttner S, Wissmann C, Jöns $T$, et al. Vascular endothelial growth factor-D and its receptor VEGFR-3: two novel independent prognostic markers in gastric adenocarcinoma. J Clin Oncol. 2006;24:228-40.

19. Suzuki S, Dobashi Y, Hatakeyama Y, et al. Clinicopathological significance of platelet-derived growth factor (PDGF)-B and vascular endothelial growth factor-A expression, PDGF receptor$\beta$ phosphorylation, and microvessel density in gastric cancer. BMC Cancer. 2010;10:659.

20. Ferrara N, Gerber HP, LeCouter J. The biology of VEGF and its receptors. Nat Med. 2003;9:669-76.

21. Ferrara N. Vascular endothelial growth factor as a target for anticancer therapy. Oncologist. 2004;9(suppl 1):2-10.

22. Jung YD, Mansfield PF, Akagi M, et al. Effects of combination anti-vascular endothelial growth factor receptor and antiepidermal growth factor receptor therapies on the growth of gastric cancer in a nude mouse model. Eur J Cancer. 2002;38:1133-40.

23. Takahashi Y, Cleary KR, Mai M, Kitadai Y, Bucana CD, Ellis LM. Significance of vessel count and vascular endothelial growth factor and its receptor (KDR) in intestinal-type gastric cancer. Clin Cancer Res. 1996;2:1679-84.

24. Tanigawa N, Amaya H, Matsumura M, Shimomatsuya T, Horiuchi T, Muraoka R, et al. Extent of tumor vascularization correlates with prognosis and hematogenous metastasis in gastric carcinomas. Cancer Res. 1996;56:2671-6. 
25. Maeda K, Kang SM, Ogawa M, Onoda N, Sawada T, Nakata B, et al. Combined analysis of vascular endothelial growth factor and platelet-derived endothelial cell growth factor expression in gastric carcinoma. Int J Cancer. 1997;74:545-50.

26. Tanigawa N, Amaya H, Matsumura M, Shimomatsuya T. Correlation between expression of vascular endothelial growth factor and tumor vascularity, and patient outcome in human gastric carcinoma. J Clin Oncol. 1997;15:826-32.

27. Maeda K, Kang SM, Onoda N, Ogawa M, Kato Y, Sawada T, et al. Vascular endothelial growth factor expression in preoperative biopsy specimens correlates with disease recurrence in patients with early gastric carcinoma. Cancer (Phila). 1999;86:566-71.

28. Potente M, Gerhardt H, Carmeliet P. Basic and therapeutic aspects of angiogenesis. Cell. 2011;146:873-87.

29. Weis SM, Cheresh DA. Tumor angiogenesis: molecular pathways and therapeutic targets. Nat Med. 2011;17:1359-70.

30. Semenza GL. Surviving ischemia: adaptive response mediated by hypoxia-inducible factor 1. J Clin Invest. 2000;106:809-12.

31. Sumiyoshi Y, Kakeji Y, Egashira A, Mizokami K, Orita H, Maehara Y. Overexpression of hypoxia-inducible factor 1alpha and $\mathrm{p} 53$ is a marker for an unfavorable prognosis in gastric cancer. Clin Cancer Res. 2006;12:5112-7.

32. Mizokami K, Kakeji Y, Oda S, Irie K, Yonemura T, Konishi F, et al. Clinicopathologic significance of hypoxia-inducible factor 1alpha overexpression in gastric carcinomas. J Surg Oncol. 2006;94:149-54.

33. Ma J, Zhang L, Ru GQ, Zhao ZS, Xu WJ. Upregulation of hypoxia inducible factor 1alpha mRNA is associated with elevated vascular endothelial growth factor expression and excessive angiogenesis and predicts a poor prognosis in gastric carcinoma. World J Gastroenterol. 2007;13:1680-6.

34. Oh SY, Kwon HC, Kim SH, Jang JS, Kim MC, Kim KH, et al. Clinicopathologic significance of HIF-1alpha, p53, and VEGF expression and preoperative serum VEGF level in gastric cancer. BMC Cancer. 2008;8:123.

35. Chen L, Shi Y, Yuan J, Han Y, Qin R, Wu Q, et al. HIF-1 alpha overexpression correlates with poor overall survival and diseasefree survival in gastric cancer patients post-gastrectomy. PLoS One. 2014;9:e90678.

36. Shah MA, Ramanathan RK, Ilson DH, Levnor A, D'Adamo D, O'Reilly E, Tse A, Trocola R, Schwartz L, Capanu M, Schwartz GK, Kelsen DP. Multicenter phase II study of irinotecan, cisplatin, and bevacizumab in patients with metastatic gastric or gastroesophageal junction adenocarcinoma. J Clin Oncol. 2006;24:5201-6.

37. Shah MA, Jhawer M, Ilson DH, Lefkowitz RA, Robinson E, Capanu M, et al. Phase II study of modified docetaxel, cisplatin, and fluorouracil with bevacizumab in patients with metastatic gastroesophageal adenocarcinoma. J Clin Oncol. 2011;29:868-74.

38. El-Rayes BF, Zalupski M, Bekai-Saab T, Heilbrun LK, Hammad N, Patel B, Urba S, Shields AF, Vaishampayan U, Dawson S, Almhanna K, Smith D, Philip PA. A phase II study of bevacizumab, oxaliplatin, and docetaxel in locally advanced and metastatic gastric and gastroesophageal junction cancers. Ann Oncol. 2010;21:1999-2004.

39. Uronis HE, Bendell JC, Altomare I, Blobe GC, Hsu SD, Morse MA, et al. A phase II study of capecitabine, oxaliplatin, and bevacizumab in the treatment of metastatic esophagogastric adenocarcinomas. Oncologist. 2013;18:271-2.

40. Ohtsu A, Shah MA, Van Cutsem E, Rha SY, Sawaki A, Park SR, et al. Bevacizumab in combination with chemotherapy as firstline therapy in advanced gastric cancer: a randomized, doubleblind, placebo-controlled phase III study. J Clin Oncol. 2011;29:3968-76.
41. Shen L, Li J, Xu J, Pan H, Dai G, Qin S, Wang L, Wang J, Yang Z, Shu Y, Xu R, Chen L, Liu Y, Yu S, Bu L, Piao Y. Bevacizumab plus capecitabine and cisplatin in Chinese patients with inoperable locally advanced or metastatic gastric or gastroesophageal junction cancer: randomized, double-blind, phase III study (AVATAR study). Gastric Cancer. 2015;18(1):168-76.

42. Okines AF, Langley RE, Thompson LC, Stenning SP, Stevenson $\mathrm{L}$, Falk S, et al. Bevacizumab with peri-operative epirubicin, cisplatin and capecitabine (ECX) in localised gastro-oesophageal adenocarcinoma: a safety report. Ann Oncol. 2013;24:702-9.

43. Spratlin JL, Cohen RB, Eadens M, et al. Phase I pharmacologic and biologic study of ramucirumab (IMC-1121B), a fully human immunoglobulin G1 monoclonal antibody targeting the vascular endothelial growth factor receptor-2. J Clin Oncol. 2010;28:780-7.

44. Lu D, Shen J, Vil MD, Zhang H, Jimenez X, Bohlen P, et al. Tailoring in vitro selection for a picomolar affinity human antibody directed against vascular endothelial growth factor receptor 2 for enhanced neutralizing activity. J Biol Chem. 2003;278:43496-507.

45. Miao HQ, Hu K, Jimenez X, Navarro E, Zhang H, Lu D, et al. Potent neutralization of VEGF biological activities with a fully human antibody Fab fragment directed against VEGF receptor 2. Biochem Biophys Res Commun. 2006;345:438-45.

46. Jung YD, Mansfield PF, Akagi M, Takeda A, Liu W, Bucana CD, et al. Effects of combination anti-vascular endothelial growth factor receptor and anti-epidermal growth factor receptor therapies on the growth of gastric cancer in a nude mouse model. Eur $\mathbf{J}$ Cancer. 2002;38:1133-40.

47. Zhu Z, Hattori K, Zhang H, Jimenez X, Ludwig DL, Dias S, et al. Inhibition of human leukemia in an animal model with human antibodies directed against vascular endothelial growth factor receptor 2. Correlation between antibody affinity and biological activity. Leukemia. 2003;17:604-11.

48. Fuchs CS, Tomasek J, Yong CJ, Dumitru F, Passalacqua R, Goswami C, Safran H, et al. Ramucirumab monotherapy for previously treated advanced gastric or gastro-oesophageal junction adenocarcinoma (REGARD): an international, randomised, multicentre, placebo-controlled, phase 3 trial. Lancet. 2014;383(9911):31-9.

49. Wilke H, Muro K, Van Cutsem E, Oh SC, Bodoky G, Shimada Y, Hironaka S, Sugimoto N, Lipatov O, Kim TY, Cunningham D, et al. Ramucirumab plus paclitaxel versus placebo plus paclitaxel in patients with previously treated advanced gastric or gastro-oesophageal junction adenocarcinoma (RAINBOW): a double-blind, randomised phase 3 trial. Lancet Oncol. 2014;15(11):1224-35.

50. Yoon HH, Bendell JC, Braiteh FS, Firdaus I, Philip AP, Cohnet LA, et al. Ramucirumab (RAM) plus FOLFOX as front-line therapy $(\mathrm{Rx})$ for advanced gastric or esophageal adenocarcinoma (GE-AC): randomized, double-blind, multicenter phase 2 trial. ASCO meeting abstracts. J Clin Oncol. 2014;32((15 suppl)):4004.

51. Lordick F. Gastrointestinal cancer. Over the RAINBOW-renaissance in antiangiogenesis. Nat Rev Clin Oncol. 2015;12(1):7-8.

52. Wilhelm SM, Adnane L, Newell P, Villanueva A, Llovet JM, Lynch M. Preclinical overview of sorafenib, a multikinase inhibitor that targets both Raf and VEGF and PDGF receptor tyrosine kinase signaling. Mol Cancer Ther. 2008;7:3129-40.

53. Sun W, Powell M, O’Dwyer PJ, Catalano P, Ansari RH, Benson AB. Phase II study of sorafenib in combination with docetaxel and cisplatin in the treatment of metastatic or advanced gastric and gastroesophageal junction adenocarcinoma: ECOG 5203. J Clin Oncol. 2010;28:2947-51.

54. Martin-Richard M, Gallego R, Pericay C, Garcia Foncillas J, Queralt B, Casado E, Barriuso J, Iranzo V, Juez I, Visa L. 
Multicenter phase II study of oxaliplatin and sorafenib in advanced gastric adenocarcinoma after failure of cisplatin and fluoropyrimidine treatment. A GEMCAD study. Invest New Drugs. 2013;31:1573-9.

55. Kang Y, Lee KH, Shen L, Yeh K, Hong YS, et al. Randomized phase II study of capecitabine and cisplatin with or without sorafenib in patients with metastatic gastric cancer: STARGATE study. Ann Oncol. 2014;25(suppl 4):iv210-53. doi:10.1093/ annonc/mdu334.

56. Bang YJ, Kang YK, Kang WK, Boku N, Chung HC, Chen JS, Doi T. Phase II study of sunitinib as second-line treatment for advanced gastric cancer. Invest New Drugs. 2011;29:1449-58.

57. Moehler M, Mueller A, Hartmann JT, Ebert MP, Al-Batran SE, Reimer P, Weihrauch M, Lordick F, Trarbach T, Biesterfeld S, Kabisch M, Wachtlin D, Galle PR, German Arbeitsgemeinschaft Internistische Onkologie (AIO). An open-label, multicentre biomarker-oriented AIO phase II trial of sunitinib for patients with chemo-refractory advanced gastric cancer. Eur $\mathrm{J}$ Cancer. 2011;47:1511-20.

58. Tian S, Quan H, Xie C, Guo H, Lü F, Xu Y, Li J, Lou L. YN968D1 is a novel and selective inhibitor of vascular endothelial growth factor receptor-2 tyrosine kinase with potent activity in vitro and in vivo. Cancer Sci. 2011;102:1374-80.

59. Wilhelm SM, Carter C, Tang L, Wilkie D, McNabola A, Rong H, Chen C, Zhang X, Vincent P, McHugh M, Cao Y, Shujath J, Gawlak S, Eveleigh D, Rowley B, Liu L, Adnane L, Lynch M, Auclair D, Taylor I, Gedrich R, Voznesensky A, Riedl B, Post LE, Bollag G, Trail PA. BAY 43-9006 exhibits broad spectrum oral antitumor activity and targets the RAF/MEK/ERK pathway and receptor tyrosine kinases involved in tumor progression and angiogenesis. Cancer Res. 2004;64:7099-109.

60. Li J, Zhao X, Chen L, Guo H, Lv F, Jia K, Yv K, Wang F, Li C, Qian J, Zheng C, Zuo Y. Safety and pharmacokinetics of novel selective vascular endothelial growth factor receptor-2 inhibitor YN968D1 in patients with advanced malignancies. BMC Cancer. 2010;5(10):529.

61. Li J, Qin S, Xu J, Guo WJ, Xiong JP, Bai Y, Sun G, Yang Y, Wang L, Xu N, Cheng Y, Zhe-Hai W, Zheng L, Tao M. A randomized, double-blind, multicenter, phase II, three-arm, placebo-control study of apatinib as third-line treatment in patients with metastatic gastric carcinoma. J Clin Oncol 2011;29(suppl):abstr 4019

62. Qin S, et al. Phase III study of apatinib in advanced gastric cancer: A randomized, double-blind, placebo-controlled trial. J Clin Oncol. 2014;32:5s (suppl; abstr 4003).

63. Lindsay CR, MacPherson IR, Cassidy J. Current status of cediranib: the rapid development of a novel anti-angiogenic therapy. Future Oncol. 2009;5:421-32.

64. Satoh T, Yamada Y, Muro K, Hayashi H, Shimada Y, Takahari D, Taku K, Nakajima TE, Shi X, Brown KH, Boku N. Phase I study of cediranib in combination with cisplatin plus fluoropyrimidine (S-1 or capecitabine) in Japanese patients with previously untreated advanced gastric cancer. Cancer Chemother Pharmacol. 2012;69:439-46.

65. TEL0805 trial: a phase 2 study of telatinib (TEL) in combination with capecitabine $(\mathrm{X})$ and cis $(\mathrm{P})$ as first-line treatment in patients (pts) with advanced gastric or gastro-esophageal junction (GEJ) cancer. A study of telatinib in combination with chemotherapy in subjects with advanced gastric cancer. Available from: http:// clinicaltrials.gov/show/NCT00952497/. Accessed 23 July 2015.

66. Pavlakis N, Sjoquist KM, Tsobanis E, Martin AJ, et al. INTEGRATE: a randomized, phase II, double-blind, placebo-controlled study of regorafenib in refractory advanced oesophagogastric cancer (AOGC): a study by the Australasian Gastrointestinal Trials Group (AGITG) - Final overall and subgroup results. J Clin Oncol (Meeting Abstracts). 2015;33(15 suppl):4003.

67. Lang SA, Gaumann A, Koehl GE, et al. Mammalian target of rapamycin is activated in human gastric cancer and serves as a target for therapy in an experimental model. Int $\mathrm{J}$ Cancer. 2007;120:1803-10.

68. Ohtsu A, Ajani JA, Bai YX, Bang YJ, Chung HC, Pan HM, Sahmoud T, Shen L, Yeh KH, Chin K, Muro K, Kim YH, Ferry D, Tebbutt NC, Al-Batran SE, Smith H, Costantini C, Rizvi S, Lebwohl D, Van Cutsem E. Everolimus for previously treated advanced gastric cancer: results of the randomized, double-blind, phase III GRANITE-1 study. J Clin Oncol. 2013;31(31):3935-43.

69. Van Cutsem E, de Haas S, Kang YK, Ohtsu A, Tebbutt NC, Ming $\mathrm{XuJ}$, et al. Bevacizumab in combination with chemotherapy as first-line therapy in advanced gastric cancer: a biomarker evaluation from the AVAGAST randomized phase III trial. J Clin Oncol. 2012;30:2119-27.

70. Aprile G, Ongaro E, Del Re M, Lutrino SE, Bonotto M, Ferrari L, Rihawi K, Cardellino GG, Pella N, Danesi R, Fasola G. Angiogenic inhibitors in gastric cancers and gastroesophageal junction carcinomas: a critical insight. Crit Rev Oncol Hematol. 2015;95(2):165-78.

71. Fontana E, Sclafani F, Cunningham D. Anti-angiogenic therapies for advanced esophago-gastric cancer. Indian J Med Paediatr Oncol. 2014;35(4):253-62.

72. De Vita F, Di Martino N, Fabozzi A, Laterza MM, Ventriglia J, Savastano B, Petrillo A, Gambardella V, Sforza V, Marano L, Auricchio A, Galizia G, Ciardiello F, Orditura M. Clinical management of advanced gastric cancer: the role of new molecular drugs. World J Gastroenterol. 2014;20(40):14537-58.

73. Cristescu R, Lee J, Nebozhyn M, Kim KM, Ting JC, Wong SS, Liu J, Yue YG, et al. Molecular analysis of gastric cancer identifies subtypes associated with distinct clinical outcomes. Nat Med. 2015;21(5):449-56.

74. Cancer Genome Atlas Research Network. Comprehensive molecular characterization of gastric adenocarcinoma. Nature (Lond). 2014;513:202-9. 